\title{
Stationary-state electronic distribution in quantum dots
}

\author{
Karel Král , ${ }^{\mathrm{a}, 1,2}$ Petr Zdeněk ${ }^{\mathrm{a}}$ \\ ${ }^{a}$ Institute of Physics, Academy of Sciences of Czech Republic, 18221 Prague 8, Na Slovance 2, Czech Republic
}

\begin{abstract}
We wish to draw an attention to a non-gibbsian behavior of zero-dimensional semiconductor nanostructures, which appears to be manifested in experiments by an effect of incomplete depopulation from electronic excited states or by an effect of up-conversion of electronic level occupation after preparing the system in the ground state of electronic excitation. In the present work the effect is interpreted with help of electron-LO-phonon interaction, which is supposed to play a role in these structures in the form of multiple-scattering of electron on the optical phonons. Quantum kinetic equation describing the process of electronic ralaxation with the inclusion of electronic multiple scattering on phonons is considered. The multiple electron scattering interpretation of the effect is supported by pointing out a considerable degree of agreement between the theoretical picture presented and a rather extensive amount of existing experimental data.
\end{abstract}

Key words: quantum dots, electron-phonon interaction, up-conversion, relaxation, statistical distribution, nanostructures PACS: 72.10.Di, 73.21.La, 73.63.Kv, 78.67.Hc

\section{Introduction}

From the point of view of mean free path of charge carriers the semiconductor nanostructures are small objects. Thanks to this size of nanostructures charge carriers can repeat their elastic scattering at the nanostructure boundaries many times and return back to a scatterer inside the nanostructure, to perform in this way coherent multiple-scattering acts. Such multiplescattering processes are not usually regarded as important in bulk semiconductor samples. In the latter, a charge carrier makes a single scattering act and leaves the target to infinity. When the scattering target in a nanostructure is the phonon system, like the

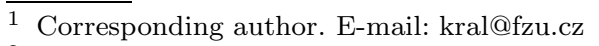
2 Support of K. K. from project AVOZ1-010-914 is acknowledged. longitudinal-optical (LO) phonons in the lattice of, say Gallium Arsenide (GaAs), the multiple scattering process can lead to a creation of multi-phonon states in the system of lattice vibrations, analogical to multi-photon states in the light of laser. Making an analogy between the multi-phonon states and the multi-photon states in the laser light, we may expect the multi-phonon states to manifest themselves as macroscopic oscillations of the whole atomic lattice of the nanostructure, making the environment, in which a charge-carrier moves, to be a system with non-conservative property from energy point of view. From such reasons we may expect unusual properties of semiconductor nanostructures, as compared to ordinary bulk sample properties.

This feature of electronic scattering on phonons in quantum dots has been recently incorporated in the description of electron relaxation theory $[1,2]$. This theory has been applied to describe the characteristics of 
electronic energy relaxation from higher energy states of electronic excitation in quantum dots $[1,2]$. Rather recently, the theory has been applied to a description of experimental effects of incomplete electronic relaxation and up-conversion of electronic level occupation in quantum dots $[3,4]$. A brief comparison of the theoretical data with experiments will be presented in this work emphasizing in this way a support which the experiments appear to provide to the multiple-scattering theoretical interpretation, postponing at this stage a more detailed analysis of the kinetic equation properties to a later work.

In addition to comparing the theoretical transport properties with experiment we perform a comparison of electronic spectral density with experiment and direct numerical computation results. In particular, measurements of optical line-shape of luminescence from exciton ground state in individual quantum dots allow us to compare with the theoretical optical line-shape properties. Besides such a comparison with experimental data, we also remark on comparison with recent numerical computation published recently in paper [5], in which electronic spectral density was computed in quantum dots numerically from definition of electronic spectral density function.

In order to outline the theory under consideration in a necessary detail, the model of electronic excitation in a quantum dot will be specified and the kinetic equation describing the relaxation process will be reminded, together with specifying the time scales which are available for the description of effects under study. Properties of the theoretical model of electronic relaxation, namely the properties of limiting stationary state distribution of electron in a quantum dot, will be characterized numerically.

In the summary we specify the implications which the theory, together with the comparison with experiments, appears to suggest with regard to statistical properties of nanostructures.

\section{Theoretical model of electronic relaxation in quantum dots}

Experiments on electronic relaxation in semiconductor quantum dots show that this process usually occurs at the time scale of picoseconds [6]. If quantum dot were not in contact with electromagnetic field, the electron-phonon system of quantum dot would achieve a stationary state within a period of picoseconds. If the state of the phonon system were kept steady, we would expect that the electronic subsystem statistical distribution equilibrates with the phonon system. In this situation we could then use the word 'stationary' for the word 'equilibrium' and vice versa. Thanks to the coupling of electronic excitations in quantum dots to electromagnetic field the above mentioned stationary state slowly decays with a lifetime, which is usually of the order of nanoseconds. With respect to the picosecond relaxation time we shall regard the time period of nanoseconds as long for achieving the stationary state due to electron-phonon interaction. At the time scale of picoseconds we shall therefore not take into account the interaction with electromagnetic field explicitly.

In a semiconductor quantum dot, or in a nanoparticle, electronic excitation may have eigenstates with energy below the outside electronic potential of this structure. In quantum dot samples the bound states may be controlled by choosing a suitable combination of two materials of such a heterostructure [7], with appropriate combination of energies of valence and conduction bands of hole and electron states. Such a heterostructure may be e. g. a GaAs qauntum dot surrounded by the material of AlGaAs [6,7]. Let us simply speak only about quantum dots in what follows, although much of our arguments may be related also to other types of nanostructures. As for the electronic states in quantum dots, we usually deal with the most simple electronic excitations, in which a single electron is present in conduction band bound states of quantum dot, while the valence band states are occupied by a single hole particle.

The electron and the hole mutually interact by electrostatic forces and both of them are coupled to lattice vibrations. The well-known differences between these two particles allows us to make a simplifying assumption about the influence of holes. Namely, it is wellknown that the holes relax in a rather fast way to their ground state and they are much heavier than the electrons. Assuming that the holes are heavy enough we completely neglect their motion, assuming that they simply contribute to the effective potential in which the electron moves. In this way the electronic excitation as a whole can be represented simply by the electron in the conduction band states of a quantum dot. For 
the sake of simplicity we then speak here about electron instead of speaking about electron-hole complex, or about an exciton.

The three-dimensional potential well, to which the electronic motion is confined when moving in the bound states, can be simply represented by the infinitely deep cubic potential well, with the lateral size $d$, in which the electron has the effective mass of the bottom of the conduction band $\Gamma$-valley of GaAs. We shall confine ourselves to the two lowest-energy electron bound states in this potential, namely to the ground state, with unperturbed energy $E_{0}$, taking $E_{0}=0$ in this work, and one of the triple-degenerate lowest-energy excited states, with energy $E_{1}$.

The single electron moving inside the quantum dot is assumed to interact with bulk modes of longitudinal optical (LO) phonons of the lattice. The electron-phonon coupling is provided by the wellknown Fröhlich's coupling [8]. The corresponding material constants will be those of GaAs crystalline material. This electron-phonon coupling is usually found to be the strongest coupling of charge carriers in polar semiconductors [9] to lattice modes. The Hamiltonian of the system can be found in the reference [4] and will be not repeated here. Throughout this work we assume that the system of LO phonons is kept at equilibrium temperature $T_{L O}$.

We shall specify the state of the electronic system by electron state occupations $N_{i}, i=0,1, N_{i}$ being mean value of electronic number operator of electrons in state $i, N_{0}+N_{1}=1 . N_{0}$ is therefore the occupation of the lower energy electronic state with energy $E_{0}$. We use the method of nonequilibrium Green's functions and apply the simple Kadanoff-Baym ansatz [10] being aware of its certain shortcomings from the point of view of causality violation [11]. We include the electronLO-phonon interaction into the electronic self-energy in the form of the self-consistent Born approximation [12]. Then we get the following quantum kinetic equation for time evolution of electronic distribution due to electron-LO-phonon interaction :

$$
\begin{aligned}
\frac{d N_{1}}{d t}= & -\frac{2 \pi}{\hbar} \alpha_{01}\left[N_{1}\left(1-N_{0}\right)\right. \\
\times & \left(\left(1+\nu_{L O}\right) \int_{-\infty}^{\infty} d E \sigma_{1}(E) \sigma_{0}\left(E-E_{L O}\right)\right. \\
& \left.+\nu_{L O} \int_{-\infty}^{\infty} d E \sigma_{1}(E) \sigma_{0}\left(E+E_{L O}\right)\right) \\
& \quad-N_{0}\left(1-N_{1}\right) \\
\times & \left(\left(1+\nu_{L O}\right) \int_{-\infty}^{\infty} d E \sigma_{0}(E) \sigma_{1}\left(E-E_{L O}\right)\right. \\
& \left.\left.\quad+\nu_{L O} \int_{-\infty}^{\infty} d E \sigma_{0}(E) \sigma_{1}\left(E+E_{L O}\right)\right)\right] .
\end{aligned}
$$

In this equation $\nu_{L O}$ is Bose-Einstein distribution function of LO phonons kept at temperature $T_{L O}$. The optical phonons are assumed dispersionless. Instead of energy-conservation delta-function, which would be present on the right-hand side of this equation in case of approximating the self-energy by bare Born approximation, the collision integral depends here on convolution of two electronic spectral densities $\sigma_{i}(E)$ corresponding to states $i=0,1$. This off-shell property of the collision integral occurs because of the presently used self-consistent Born approximation, which, as said above, can be seen as introducing effectively virtual multi-phonon states together with a certain effective energy non-conservativeness of the system in the present system of electron-phonon dynamics. Because of the significant property of quantum dots, due to which electronic motion is severely restricted in space in all three dimensions, the self-consistent Born approximation, together with the off-shell property of the collision integral, may appear to be substantial for describing processes in quantum dots.

The electronic spectral densities are determined with help of electronic Green's functions, which are again given by the electronic self-energy. The selfconsistent Born approximation to the electronic selfenergy is given by the following self-consistent equation for retarded self-energy $M_{n}^{R}(E)$, in which $G_{m}^{R}(E)$ is retarded electronic Greens's function in state $m$ :

$M_{n}^{R}(E)$

$$
\begin{aligned}
= & \sum_{m} \alpha_{n m}\left[\left(1-N_{m}+\nu_{L O}\right) G_{m}^{R}\left(E-E_{L O}\right)\right. \\
& \left.+\left(N_{m}+\nu_{L O}\right) G_{m}^{R}\left(E+E_{L O}\right)\right] .
\end{aligned}
$$

Despite the fact that the unperturbed Hamiltonian does not contain any continua in the spectrum of its 


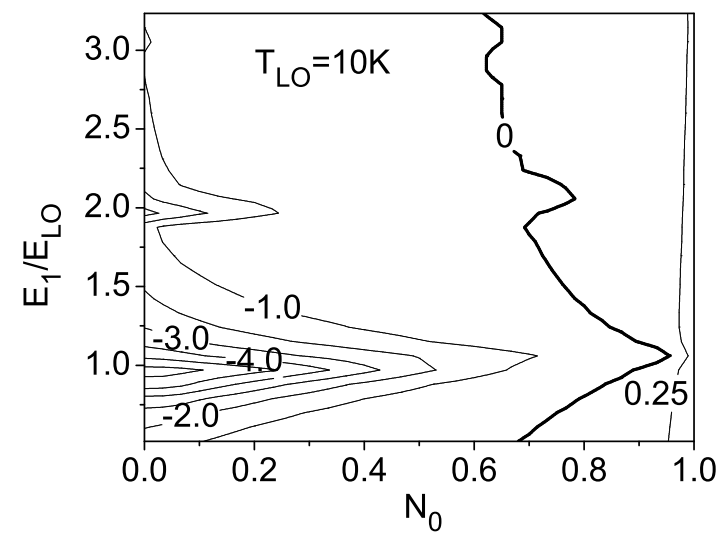

Fig. 1. Contour plot of relaxation rate $d N_{1} / d t$ in units of $\mathrm{ps}^{-1}$. Note the curve with index 0 marking such detuning $E_{1} / E_{L O}$ and occupation $N_{0}$ at which relaxation rate is zero at the given temperature of lattice.

eigenstates, the solution of (2) taken to the limit of infinite number of iterations of the equation gives electronic spectral densities which are not completely discrete. This property was demonstrated earlier [12]. In an intuitive way, this property of the solution can be understood on the basis of multiple scattering motion of electron in a quantum dot and a presence of virtual multi-phonon states. Parameters $\alpha_{n m}$ determine the electron-phonon coupling. The reader is referred e. g. to paper [4] and to references cited in.

Earlier numerical evaluations of the relaxation rate $d N_{1} / d t(1)$ show that the electron relaxes quickly on picosecond time scale without any strong dependence on the detuning $E_{1} / E_{L O}$ between the electron energy level separation and the energy of the optical phonon $[1,2]$. On the basis of standard experience we might expect that when the electron is prepared in the higher-energy state $n=1$, with energy $E_{1}$, the electron-phonon interaction will at the end lead to the limiting occupation $N_{0}=1$, at zero temperature of lattice. However, the above given relaxation rate formula and, as will be shown in the next section, a number of recent experiments too, do not seem to provide such a simple picture of electron energy relaxation in quantum dots. We shall comment on this question in what follows, adding also a brief review of experiments in the next section.

Let us characterize first the solution of relaxation rate equation $(1,2)$ for some special situations. Let us consider the limiting state at which electronic distribution reaches stationary distribution at a given temper-

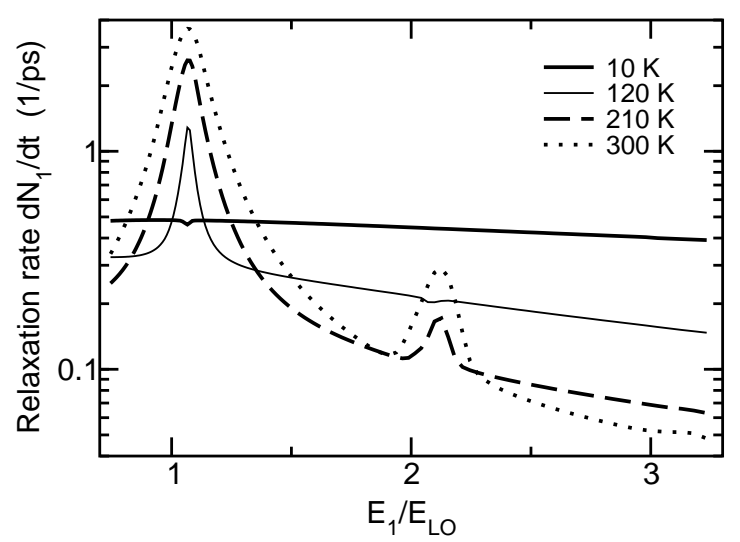

Fig. 2. Rate $d N_{1} / d t$ of up-conversion of electronic occupation from the fully occupied lower energy state $\left(N_{0}=1\right)$ to the upper energy state.

ature $T_{L O}$ of the lattice. This state can be obtained by letting evolve in time the electron distribution according to the differential equation $(1,2)$, or simply evaluating the relaxation rate, finding out, for given conditions, when this quantity happens to be zero. In Fig. 1 we present a contour plot of the relaxation rate $d N_{1} / d t$ as it depends on the detuning $E_{1} / E_{L O}$ and occupation $N_{0}$. The curve marked with index 0 displays conditions under which the relaxation rate is zero. Therefore, this curve gives the stationary states to which the system evolves in the limit of long time. We see that in some dependence on the detuning the stationary value of the occupation $N_{0}$ of the lower energy state is about 0.7 , with a certain strong extremum near the detuning equal to 1.

When the electron is prepared in the low energy state at the beginning of evolution under electron-phonon interaction, the relaxation process displays a tendency to increase the occupation of the upper energy state. Detailed calculation of the evolution of the system shows [3] that this process occurs on the time scale of picoseconds. The relaxation rate describing this process of upconversion in the state when $N_{0}=1$ is presented in Fig. 2. In this figure the rate of up-conversion displays resonance structure at large temperatures of lattice, while the rate is nearly structureless in the limit of zero temperature of the lattice. The effect of nonzero rate of up-conversion near $T_{L O}=0$ can be explained by the nature of the electron-phonon interaction, which leads to multiple-phonon states in the quantum dot, which leads to an non-conservativeness of the effective Hamil- 
tonian in which the electron moves. Technically, the term, which is responsible in formula (1) for this zero temperature up-conversion, is that proportional to the factor $N_{0}\left(1-N_{1}\right)\left(1+\nu_{L O}\right)$. This term corresponds to an emission of phonons in this process. We give the same explanation of the plausibility of the simultaneous electronic up-conversion and phonon emission: because of the multiple-scattering and the off-shell nature of the process we do not control the conservation of energy in formula (1). Because of the off-shell property of the multiple-scattering electron-phonon scattering processes, the comparison of unperturbed energies of electrons and phonons may not give us here a sufficient control over energy balance in the present model and approximation. Obviously, this point deserves an attention in future work.

In Fig. (3) we show the limiting stationary occupation $N_{0}$ of the lower energy state calculated from kinetic equation $(1,2)$, as a function of the lattice temperature $T_{L O}$. We see that the amount of electronic density staying in the low energy state is much lower than the amount given by Fermi-Dirac distribution at the same temperature $T_{L O}$ of lattice. This effect may be interpreted as a non-gibbsian behavior of electronphonon system in semiconductor quantum dots. This effect is obtained within our present simple theoretical model with the present simple approximations to electronic self-energy. The reader is referred to papers $[3,4]$ for other numerical results on the topic of time dependence of electronic relaxation in quantum dots.

The model and the kinetic equation deserve themselves a further theoretical analysis, which should test e. g. the stability of the effect with respect to further extending the model of quantum dot towards a sufficiently realistic model of quantum dot, and also with respect to improvements of the approximations leading to the kinetic equation. In particular, we would like to gain a better understanding of the energy balance in processes under consideration. Instead of proceeding further in this respect we give way in this work to presenting a summary of the present state of agreement between the theory and the available experimental data.

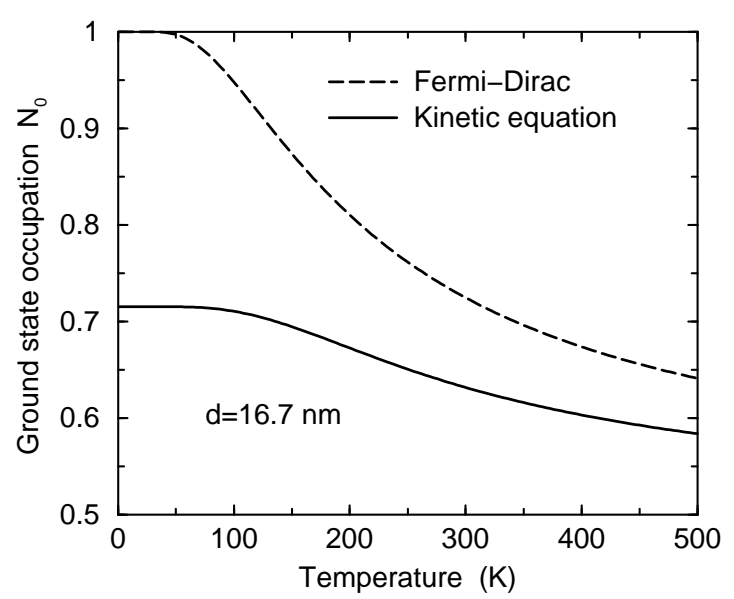

Fig. 3. Fermi-Dirac occupation $N_{0}$ of lower energy state in quantum dot with lateral size of $d=16.7 \mathrm{~nm}$ (dashed). Full line gives occupation $N_{0}$ calculated from the kinetic equation in steady state.

\section{Experiments on electronic relaxation}

The theoretical value of the relaxation rate, given by $(1,2)$ and being often in the range of $1 / \mathrm{ps}$, agrees well by the order of magnitude with a large number of experimental measurements on the electron energy relaxation in quantum dots (see e. g. [6]). In addition to this, the relaxation rate depends to some extent on the detuning $E_{1} / E_{L O}$, giving resonance spikes on the relaxation rate (1) at the detuning approximately equal to an integer [1]. The experimental data on photoluminescence excitation (PLE) display often a certain resonance structure with a periodicity equal to optical phonon energy along the photon energy variable axis $[13,14]$. The presence of the resonance structure in the electronic relaxation rate is in agreement with the purely optical experimental method used sometimes for detection of the presence of quantum dots in the grown samples (see ref. [14], section 3.3). The experimental electron relaxation rate and the resonance structure can be related to recent theoretical calculations of the electron energy relaxation in quantum dots based development of polaron concept in quantum dots and on considering finite lifetime of optical phonons (e. g. [15]). This latter approach, in which the question of polaron on quantum dots has been developed considerably, seems to give agreement with experiment in certain restricted intervals of the magnitude of the de- 
tuning. The up-conversion effect has not been reported in the polaron approach as yet.

The electronic spectral density itself, as it is given by the self-consistent equation (2), gives, at integer level occupation, the spectral line shape having the functional dependence of the type $1 / \sqrt{(} E)$ at low temperatures [12], which has zero width at $T_{L O}=0$ in the sense of full width at half maximum. The temperature dependence of the main feature of the spectral density [16] is found in good agreement with experiments [17]. The electronic spectral density line shape profile of the lower energy electronic state in our model, regarded to correspond to the optical line shape of the ground state electronic excitation in the quantum dot, has a shoulder on the lower energy side, with the shoulder width of the order of milielectronvolts. Such a characteristic line-shape of the lowest energy optical transition has also been recently detected in single-dot experiments on polar semiconductor quantum dots $[18,19]$ and on Si nanoparticles [20].

Still another supporting argument is provided to the presently used self-consistent Born approximation to the self-energy by the recently published numerical analysis based upon taking into account the electronLO-phonon coupling in numerical evaluation of the electronic spectral density from definition [5]. The numerical results of this study, although they cannot provide continuous spectral density because the calculation is based on the phonon Fock's space with only finite number of phonons included, avoiding any inclusion of coherent states of the phonon modes, gives a structure of discrete lines of the spectral density, the envelope of which nevertheless appears to remind interestingly the results of the presently used self-consistent Born approximation. This remarkable agreement of the spectral density with experimental data, and even with results of direct numerical analysis, supports the plausibility of choosing self-consistent Born approximation to include the electron-LO-phonon coupling in the present transport equation.

Let us turn our attention now to experiments related to the electronic transport measurements of effects like the electronic up-conversion in quantum dots.

There is a relatively large number of experiments on anti-Stokes emission and electronic up-conversion in nanostructures and other systems in scientific literature. Some of them were mentioned in papers $[3,4]$ and some will be summarized elsewhere. Here we pay attention to the following ones. In the experimental papers which we want to mention the measurement of luminescence provides data, which can be related to occupation of bound states of the electronic system in quantum dots.

When we have in mind excited state of an exciton in a quantum dot, we shall speak about excited state. We shall speak about ground state, when having in mind the lowest energy state of electronic excitation in the dot (an exciton). One of the most interesting papers on incomplete electronic level depopulation is the measurement of time resolved luminescence signal $[21,22]$, measured at 6 or at $40 \mathrm{~K}$ on InGaAs quantum dots, where depopulation of an excited state of electronic excitation in quantum dots is determined. It is shown that within picoseconds the excited state is being depopulated, but the depopulation process stops, leaving the depopulation of excited state incomplete. The amount of excited state population at which the depopulation stops is in the order of tens of percents. Similarly such process of incomplete depopulation of excited state was detected in reference [23], in which the authors measured the same process at $290 \mathrm{~K}$ in InAs/GaAs quantum dots. After exciting the exciton ground state, they report appearance of emission from excited state of exciton, which is detected about 60 ps after excitation of the ground state. The authors regard the process of appearance of the excited state luminescence as unusually fast for to be caused by simply a thermal population of the exciton excited state. According to their measurement, the luminescence from the resonantly excited ground state decreases quickly on a picosecond scale, with the ground state signal becoming weaker by several tens of percents, after which the excitation decays on the scale of nanoseconds. Let us emphasize that the remarkable fast process of partial depopulation of the resonantly excited exciton ground state can be explained by the up-conversion process provided by the present theory.

Similarly an incomplete depopulation has been detected in CdSe quantum dots at $300 \mathrm{~K}$ [24]. The authors also report their observation about a certain difficulty to achieve in their experiment a state with an integer occupation of quantum dot levels. The integer level population is unstable even within the present theory.

Diener et al. measured anti-Stokes luminescence at $4 \mathrm{~K}$ in coupled Si nanocrystals [25]. The work puts em- 
phasis on the excitation intensity dependence of the up-converted emission signal. Although the measured effect is interpreted in terms of Auger mechanism of upconversion of electronic system to excited states, the data presented do not seem to display any strong excitation intensity dependence, leaving space for an alternative interpretation too. Similarly, measurements of emission from excited states was reported in the reference [26]. The authors also have put emphasis on excitation intensity dependence of excited state luminescence spectrum. Although a factor of 90 in variation of excitation intensity did not seem to influence the spectra significantly enough, the experiment was interpreted in terms of Auger mechanism of luminescence up-conversion. In a quite similar way, excitation intensity dependence of the excited states luminescence was measured some time ago without showing a strong appearance of such a dependence in experiment on GaAs/AlGaAs quantum dots [27]. Also this measurement was interpreted in terms of Auger mechanism of electronic state occupation up-conversion. In experimental paper reported in reference [28] the effect of upconversion is nicely demonstrated in the low excitation intensity limit in InGaAs/GaAs quantum dot systems.

Even the effect of the up-conversion to wetting-layer states has been detected, using photoluminescence and photoluminescence excitation measurement [29]. Let us remark at this point that recently we have given theoretical arguments in favor of existence of such an effect in dense quantum dot samples excited to a sufficiently large degree of electronic excitation [3].

Let us also remark that at the low level of electronic excitation in luminescence measurements the present multiple-LO-phonon scattering mechanism may be at least a suitable alternative to the Auger mechanism of the up-conversion, while at larger excitation intensities, under which the Auger mechanism becomes possible at all, the phonon mechanism may be at least contributing to the up-conversion mechanism. A more detailed relation between the role of the two mechanisms of up-conversion has not yet been shown.

\section{Summary}

In the experiments which we mentioned above the electronic subsystem displays a tendency to reach a state with a non-integer occupation of electronic states, even in the limit of zero temperature of lattice. The numerical results presented here display this tendency as well. The experiments also show that the up-conversion effect can be observed even at low intensity of electronic excitation of quantum dot sample, which restricts somewhat the interpretative potential of Auger mechanism in the present context. Besides the agreement of the magnitude of electron energy relaxation rate with experiment, also the presence of resonant features of the relaxation rate dependence on level detuning appears in correlation with the earlier reported practical method of detection quantum dots in a sample. Also the computed line shape and shoulder width compares well with experimental and other numerical data. Finally, the luminescence experiments on incomplete depopulation and up-conversion appear to agree well with the trends indicated in multiple-LO-phonon scattering mechanism numerical results in quantum dots.

The above presented relation between the theoretical and the experimental data provides supporting arguments in favor of the presently discussed theoretical interpretation of the effects. Undoubtedly, further experiments are needed in order to delineate more clearly the validity of the present model and approximations to the kinetic equation for electronic relaxation in quantum dots, including the energy balance question. Nevertheless, providing that the mechanism presented here will show up as a valid mechanism of electron-phonon motion in quantum dots, these systems might represent a candidate for the practical realization of a nongibbsian behavior of a small system in steady state. At the same time, even without speaking about a need to understand nanostructures for the purpose of applications, this small quantum mechanical system might be an interesting object for further study of nonequilibrium processes and statistical physics in small interacting systems.

In particular, the present relation between theory and experiment leads to the conclusion that the steady state electronic distribution, which may be achieved within picoseconds in nanostructures, is not given by Gibbs distribution with the temperature equal to the temperature of the lattice, but it should instead be determined by a solution of a kinetic equation. In the present case of two-level electronic system such a solution would then mean that the electronic temperature 
would not be necessarily equal to that of the lattice modes with which the electrons interact in this nanostructure. Beyond such a behavior of the system there would then be a smallness of the system with respect to the dephasing mean free path of electron, which conditions would make situation open for an off-shell behavior of multiple scattering of electrons. Because of some degree of carrier confinement present also in other nanostructures, certain effects of a similar nature should not be unexpected in them.

The volume of agreement between the present theoretical scheme and experimental experience is rather broad, so that it may seem that a mere accidental coincidence between theory and experiment is not likely. At the same time questions may be arisen both about the theory and the experiment. The behavior of charge carriers in transport processes in quantum dots therefore deserves a further attention.

\section{References}

[1] K. Král et al., Phys. Status Solidi B 208 (1998) R5-R6.

[2] H. Tsuchiya et al., J. Appl. Phys. 832574 (1998).

[3] K. Král et al., Nanotechnology, IEEE Transactions on 3, (2004) 17-25.

[4] K. Král et al., Surf. Sci. 566-568 (2004) 321-326.

[5] M. I. Vasilevskiy et al., Phys. Rev. B 70 (2004) 035318.

[6] A. J. Nozik, Annu. Rev. Phys. Chem. 52 (2001) 193.

[7] A. D. Yoffe, Adv. Phys. 50 (2001) 1.

[8] J. Callaway, Quantum theory of the solid state, Part B, Academic Press, New York, 1974.

[9] C. Jacoboni et al., Rev. Mod. Phys. 55 (1983) 645.

[10] L. P. Kadanoff and G. Baym, Quantum statistical mechanics, W. A. Benjamin, Inc., New York, 1962.

[11] H. Haug, S. W. Koch, Quantum theory of the optical and electronic properties of semiconductors, 3rd ed., World Scientific, Singapore, 1998.

[12] K. Král et al., Phys. Rev. B 57 (1998) R2061.

[13] N. N. Ledentsov, Semiconductors 33 (1999) 946.

[14] N. N. Ledentsov et al., Semiconductors 32 (1998) 343.

[15] O. Verzelen et al., Physica B 316-317 (2002) 1-7.

[16] K. Král et al., Physica B 272 (1999) 15-17.

[17] K. Ota et al., Physica E 2 (1998) 573-577.

[18] E. Pelucchi et al., Phys. Stat. Sol. B 238 (2003) 233236.
[19] R. A. Taylor et al., Physica E 21 (2004) 285-289.

[20] I. Sygurov et al., submitted to Phys Rev. Lett.

[21] J. Urayama et al., Phys. Rev. Lett. 86 (2001) 4930.

[22] J. Urayama et al., Physica B 316-317 (2002) 74-80.

[23] F. Quochi et al., Physica B 314 (2002) 263-267.

[24] P. Guyot-Sionnest et al., Phys. Rev. B 60 (1999) R2181-R2184.

[25] J. Diener et al., Optical Materials 17 (2001) 135-139.

[26] K. Ikeda et al., J. Lumin. 108 (2004) 273-276.

[27] U. Bockelmann et al., Phys. Rev. B 55 (1997) 44564468.

[28] L. Zhang et al., Appl. Phys. Lett. 79 (2001) 3320.

[29] C. Kammerer et al., Phys. Rev. Lett. 87 (2001) 207401. 